\title{
Addressing the
}

backlog of patients:

\section{A view from across}

\section{the profession}

\section{David Westgarth}

Editor, BDJ in Practice

\section{Introduction}

'Oh sir... it's only wafer thin'.

The words of the Maitre D' to Mr Creosote in Monty Python's Meaning of Life, just as he wraps up his dinner. Of course, Mr Creosote goes on to protest he is too full, unable to even accept one wafer thin after dinner mint, before finally accepting it and quite literally exploding.

Now, let me be clear: not for one minute am I suggesting dentistry in the UK is a man addicted to eating and drinking whatever he wants, as amusing as that may seem. What I wonder is whether dentistry is bloated, ready to blow, completely overwhelmed by the backlog created over a number of years.

Across England, Wales, Scotland and Northern Ireland you find a watermark of similarities amongst a number of unique differences. This is the same story when it comes to high street dentistry - both NHS and private - hospitals and community dental services. The question is just how bad is the backlog of patients, and what can be done about it?

\section{Is there a backlog?}

Shawn Charlwood, Chair of the British Dental Association's General Dental

\section{Key points}

$\rightarrow$ Pre-pandemic issues were bad; exacerbated by COVID-19

$\rightarrow$ High street, private, hospitals and community settings affected in different ways

$\rightarrow$ How can these issues be addressed?

Practice Committee, reports a worrying and deteriorating situation.

'We have seen a significant increase in the level of patient treatment backlog in all four nations of the UK during the last 18 months', he said. 'The pandemic and the associated infection control protection restrictions, particularly fallow time, have resulted in an increased backlog affecting all regions and both adults and children. More than 30 million NHS courses of treatment have been lost since the first lockdown and patients are presenting with more advanced disease patterns, requiring more extensive treatment and intervention. This pattern of disease presentation applies to dental 
caries and periodontal disease but also to the oral malignancies, with the serious consequences of late presentation and diagnosis.'

Paul Mottram, Chair of the British Dental Association's Private Practice Sub-committee, also highlighted capacity issues.

'For dental practices who provided the majority of their practice income treating patients on a private basis, the backlog was worked on immediately as their livelihoods depended on throughput in their practices, particularly considering practitioners were unable to use the SEISS scheme and practice owners had overheads to meet.

'There has been a reduction in chair time capacity due to the IPC guidelines and the fallow period and thus output although practices are open as per their pre-pandemic hours. There are an increasing number of such practices who have had to cease accepting new private patients as they are overwhelmed with their own cohort of patients and have no capacity to see additional private patients.'

Peter Dyer, Chair of the British Dental Association's Central Committee for Hospital Dental Services, said the backlog remains a major topic of discussion - particularly oral malignancies Shawn alluded to.

'It's an issue that is very much at the forefront of our minds as it is dominating the environment and culture we work in. Primary care is very much the gatekeeper of what happens in secondary care, and we're feeling the knock-on effects of the problems the high-street is facing.

'Compared to the issues we've seen within other areas of the profession, there are two main areas we've been able to keep going, and that is prioritising trauma and cancer cases. Trauma cases haven't changed a great deal - we see those patients there and then, but theatre availability and bed capacity have been affected largely due to staff re-deployments to treat COVID-19 patients. If hospitalisations begin to increase again, this will clearly come under pressure so must be accounted for in any plans the government has.

'My main concern is that we've seen fewer cancer patients as a result of COVID-19, but at the moment there is no definitive data to say to what extent. We know early diagnosis is crucial, and I fear we've not seen anywhere near as many patients as we should have.'

Charlotte Waite, Chair of the British Dental Association's England Community Dental Services Committee, added the situation was more complex than it suggests. 
'There is no simple answer to this I am afraid. The backlogs affecting CDS vary significantly across the NHS regions. The factors which will influence the restoration and recovery of CDS are unique to each and every provider organisation and so when looking to understand the backlogs in CDS we must keep this in mind.

'For example, some services are still providing support to Urgent Dental Care Sites and almost every CDS provider in England supported these services during the pandemic, which may have had an impact on their service delivery.

'CDS moved to restoration and recovery at different times and at different paces. Vulnerability of our patient cohort, infection prevention control and social distancing measures have all significantly reduced capacity.

'Prioritisation of existing patients, as well as those on waiting lists has taken up significant time of the workforce. Urgent cases are being prioritised by GDPs and so many of the patients referred are in this category, again adding to pressure on service. Many of our existing patients would be deemed vulnerable and by following

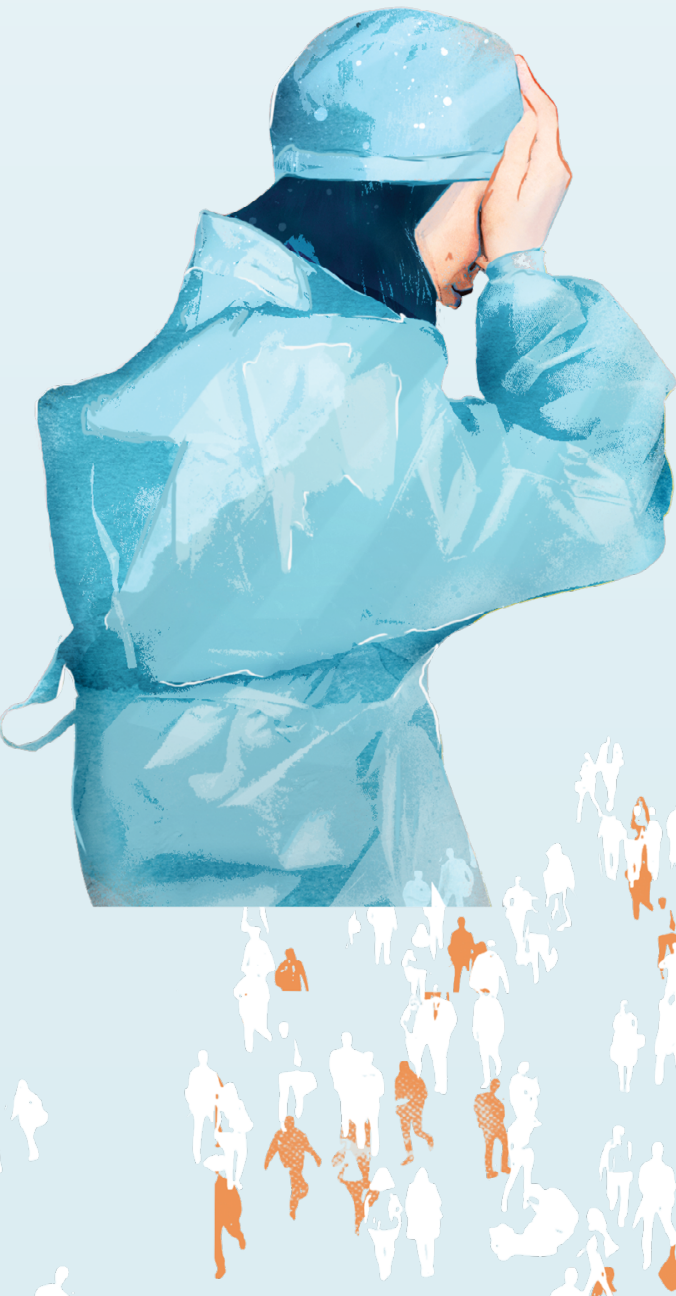

the current national standard operating procedure, they should also be seen as a priority too.

'The way that referrals are received and triaged varies across CDS and therefore data collection around waiting lists and backlogs is difficult to interpret from service to service. However, NHS England and Improvement regional dental commissioning teams, are now collecting waiting list data from all CDS in a standardised way. Collecting these data will provide a national picture of waiting times across CDS. I understand that the data collected will feed into NHS England and NHS Improvement's wider programme of work looking at the backlog of care and the restoration of services.'

Nikki Patel, Chair of the British Dental Association's Young Dentist Committee, painted a slightly different picture.

'From my perspective and bearing in mind there are large variations between England, Wales, Northern Ireland and Scotland, the backlog of patients waiting to be treated is more akin to high levels of built-up demand. Yes, there are long waiting lists for NHS and private work - in my practice there's a waiting list for treatment, particularly on the small private list and from talking to others this isn't uncommon.'

\section{New problems, or compounding of old ones?}

Much of the discussion I have had with those in the profession centres around what it was like before the pandemic took hold in the UK. There is an immediacy bias to be wary of - since March 2020 practitioners have been under and through a significant period of stress - and so many refer to PPE. After all, the needs of the patient haven't changed, there are just more of them.

I asked Peter whether today's issues are solely due to the pandemic or whether they were already in the foreground.

'Although some Trusts have dedicated trauma units and some don't, which can determine how quickly a patient is operated upon, in large it was an area that remained relatively free of some of the bureaucracy seen in other areas of hospital dentistry. Patients could move through the system provided there were adequate numbers of hospital beds and there was theatre availability. Were they improving? I would say they weren't worsening and not necessarily a result of the pandemic.

'The same can't be said for oral cancer. The two-week referral pathway was certainly being squeezed pre-pandemic. Understandably primary care practitioners were being safe rather than sorry, and we'd see large numbers of patients, not all of whom would go on to be diagnosed with oral cancer. The diagnosis stage is where we started to see a bottleneck effect. Once a diagnosis was made, patients would then leave or pass through the system. Intensive care bed availability wasn't necessarily improving, and it goes without saying this was largely non-existent at the height of the pandemic.

'Fundamentally, more needs to be done to get the message out there about oral cancer. That's not a pandemic-specific issue - it's been an issue for as long as I can remember. Yet, with issues accessing primary care, the belief that 'it's nothing, it'll go away' and that it's a disease that affects the poorer areas of society, there are significant challenges ahead. Better awareness - and a bigger focus on all areas of prevention - could stop backlogs from building up in the first place, especially at a time when funding is being squeezed like never before.'

According to Charlotte, there was already a historic level of need.

'CDS services have often been commissioned to meet a historic level of need for the population they serve', she said.

'The BDA has called for robust needs assessment, to be part of all commissioning processes to ensure that all services are commissioned appropriately to meet the needs of that population. For example, research relating to the level 
of commissioned domiciliary dental services, by the BDA in 2019 , suggested that only $1 \%$ of the dentistry required to meet the needs of this population was being commissioned. With an ageing population this must be addressed moving forward.

We have also called for equitable access for our patients to specialists, particularly in paediatric and special care dentistry. Access to specialist led services remains a post code lottery and is contributing to health inequalities. Training at all levels needs to be embedded and appropriately funded within the CDS, from foundation and core training, through to specialty training.

Recruitment and retention is also a significant concern, with an ageing workforce, there is real concern that the numbers of CDS dentists will decline year on year.'

Recruitment and retention are issues Nikki pointed to as factors not solely due to COVID-19.

'There is no denying parts of the country have no access to NHS dentists and waiting lists are atrocious, and the pressure young dentists work under is getting worse, but the balance between seeing patients we needed to and patients we can do has shifted, she told me.

'More and more discussions amongst young dentists I and the committee represent centres around their desire to reduce their commitment to NHS work and moved to mixed and purely private work. This was a huge issue prepandemic and has only moved towards more handing back their NHS contracts. Lower pay, low morale, a lack of flexibility, no incentive to upskill and the pandemic on top has just pushed too many away from the service, and it's not a surprise. There are many younger dentists who will be entering the system having graduated who accept the pay and conditions because it's all they know, and gradually you see many realising how different things can be than they anticipated - the alternative is so much more appealing. We can't sweep these issues under the carpet blaming COVID-19 - they have been with us for a while.'

Shawn agreed that morale has been a longstanding issue.

'The situation for NHS dentistry was deteriorating pre-COVID-19 with NHS dental services only being funded and commissioned for approximately half of the population. Significant numbers of patients were struggling to access NHS dental services pre-COVID-19, which generated plenty of media headlines and letters to local MPs.

'As Nikki suggests, there were increasing numbers of practices that were struggling to retain dentists within the NHS dental service and many practices were failing to successfully recruit and fill vacancies. Extensive surveys by the BDA showed a low level of satisfaction and morale for dentists working within the UDA system with low morale levels directly proportionate to the level of NHS commitment.'

Recruitment was just one of the issues the private sector faced, according to Paul.

\section{'We have also called for equitable access for our patients to specialists, particularly in paediatric and special care dentistry. Access to specialist led services remains a post code lottery and is contributing to health inequalities.'}

'A large number of private practices were experiencing issues training and recruiting business managers, practice managers and treatment co-ordinators who would fit into their business model', he added. 'Digital dentistry was in the early adoption phase by the private sector. The sector also had to contend with was the increasing corporate presence in the profession and individual practices had to differentiate themselves from their competitors without reducing their pricing structure, namely optimal customer care experience being essential.

'I also echo what Peter added earlier regarding late presentations of oral cancer. In our own practice since reopening in June 2020 we have seen two new patients who after examination and assessment were urgently referred into secondary care hospital services and had squamous cell carcinomas confirmed. Both had delayed presentation to the practice for four months each, the most recent one being last month. Both patients mentioned they thought the symptoms would go away and were fearful of the pandemic with the most recent patient thinking it was a dental infection. I am very concerned about these incidences being replicated across all the nations as I practice in a ward of lower deprivation compared to the other regions in the UK'

\section{The situation today}

A relatively grim picture across the board, one would suggest. The old saying of 'sometimes things have to get worse before they get better' perhaps applies to the profession, so I asked what these prepandemic issues were like today, and were they getting worse or improving.

'There are an increasing number of reports of practices experiencing loss of key staff members in managerial roles to other local practices via headhunting, Paul said. 'This means these roles have become difficult to fill with the appropriate skillset on the immediate basis practices need to recruit. The reduction in all dental staff personnel on the GDC register has meant recruitment is of acute concern across the country, particularly dental nurses. This has driven pay rates upwards above inflation.

'A positive has been the advancement of digital dentistry. It has moved into the early majority phase and patients are visualising the benefits that this can provide, and this will only improve with time as technology adapts.'

The situation isn't any better for their general dental practice colleagues, according to Shawn.

'The situation has significantly worsened during the pandemic period for many practices', he said. 'The poor position has been accentuated and COVID-19 has highlighted the inadequate level of commissioning of NHS dental services and how unsustainable the current UDA system is.

'The result is the BDA receiving more calls from members asking how they hand back their NHS contracts, which is not at all surprising given the challenges that practices have faced during the pandemic but also the failure by successive governments to address reform of the failed UDA contract.

'The continued frustration and concern about the UDA system has been exacerbated by COVID-19 and what we are seeing now is a deteriorating picture in terms of recruitment and retention of NHS dentists but other team members as well.

'Practice owners are seeing significant increases in the cost of materials, disposables and utilities as well as a competitive job market and are considering the viability of their practices within the NHS. Younger colleagues are increasingly seeing their future outside the NHS, where they have the time and resource to practice dentistry as they have been taught at dental school.

'Like others have pointed out, recruitment and retention problems are significant issues and are no longer apply exclusively to rural or coastal communities and we are now seeing reports of difficulties throughout all regions of the UK. The headlines of DIY dentistry by patients have become the new normal, a completely unacceptable state of affairs 
in a modern $21^{\text {st }}$ century economy which advocates health care available to all.'

While Nikki agreed the UDA system is forcing dentists out of the NHS, she questions whether this is choice or necessity.

'Shawn is absolutely right about the UDA system, but I'm not sure young dentists really have a choice', she explained. 'If pay and inflation continues on the trajectory it is, it will be impossible to maintain the quality of life needed to make NHS dentistry an attractive career prospect. When you come out of university and straight into a job that pays somewhere in the region of $£ 30,000$ the jump from nothing to that basic is appealing, but from my position as YDC chair, once you're qualified for a handful of years, there's a bit of a realisation of the bigger picture.

'The narrative here needs to change. No-one envisages when they start out that they'd willingly hand back their NHS commitments - the NHS is a wonderful and truly unique service. It's more a question of they feel there is no choice but to do so, and in almost all cases begrudgingly so.'

Peter believes the pandemic has firmly pushed many of these issues under the microscope.

'The word I'd used is magnified', he said 'All the small things that were once barely manageable are now on the wrong side of that assessment. We are still seeing referrals, but that's dependent on GPs actually seeing patients and GDP colleagues making the right referral at the right time. With the access problems mentioned by others, it's a problem. We're still prioritising both of the areas I've discussed, but the number of patients we're seeing is down and still we're seeing queues for trauma and oral cancer patients, which is unacceptable. On top of that there's a huge backlog of extractions, and you can't see two patients at once using the same theatre space.'

According to Charlotte, we're still some way off addressing the problems that mounted up since March of last year.

'With winter pressures and the impact of COVID-19 still being felt, the recovery phase continues and we are in no way back to where we were pre pandemic.

'While we're still mindful of COVID-19, we're still seeing some vulnerable patient groups a little hesitant to engage with healthcare services, which is understandable given the associated levels of anxiety. Time will tell if this improves throughout the winter, and it will be important for research to take place on this moving forward so that we can understand how we can best support these patient groups.
'It may be that some patients become 'lost' within the system and the reasons for this, will need to be explored and understood in order to plan services to meet the needs of our patients. In medicine we are already seeing a call for the development of 'community hubs' which will take diagnostic services out of hospitals and out to patients in primary care. Due to the cohorts of patients seen within the CDS, many of them will rely on other people to arrange appointments and provide support to bring them to their dental appointments. CDS will have a role in advocating for these patients and ensuring they have the support they need to access services - no-one should be left behind.

'The extra pressure this places on those working within the community is a stark reminder that the welfare of an often exhausted workforce will need to be safe guarded. As a profession we often don't reach out for help when we need it. I believe the impact of pandemic on our workforce may be felt for years to come. Supporting our existing workforce, allowing flexibility and listening to their needs will be crucial.'

\section{'What is patently obvious is that dentistry is in a difficult place. As ever, there are often opportunities that come out of difficult circumstances, so what opportunities does dentistry have to improve services across the board?'}

\section{Improving the situation: what can be done now?}

What is patently obvious is that dentistry is in a difficult place. As ever, there are often opportunities that come out of difficult circumstances, so what opportunities does dentistry have to improve services across the board?

'I believe that the NHS Long Term Plan provides an opportunity for community dental services to work within the Integrated Care Systems and develop collaborative relationships, which will benefit our patients and our services and allow for more equitable access for our patients', Charlotte suggested.

'In order to achieve this, dentistry will need to have a strong voice and be able to influence the ICS agenda. This will be particularly important for CDS who I think could benefit significantly from closer working with other health and social care providers.
'Networking and collaboration take time and clinicians will need to be supported and given time to shape this work, as the new ICS structures evolve. The BDA will continue to lobby to ensure that dentistry has a voice within the ICS structures. We must also ensure that community dentistry is engaged with by all ICS and not as part of a postcode lottery.

'Digital transformation of the NHS has made the headlines recently. Access to summary care records for all CDS services is something which the BDA has advocated for in the past and although some services do have access, it isn't the same across the board. Access to summary care records has the potential to improve patient safety and remove bureaucracy both for the dentist and the general medical practitioner and lead to less delays in treatment.'

Peter also mentions the Integrated Care Systems, but sounded a warning.

'We really need to be aware of the apparent move to privatise large areas of dentistry by stealth under the auspices of the Health and Care Bill,' he said. 'The principle of funding primary, secondary and social care together through Integrated Care Systems is in principle a good one, but smaller dental departments will eventually come under threat of closure or being swallowed up by larger Trusts covering a wide area, again affecting access.

'We'd be facing a scenario where there's an artificial divide where elective treatment is moved to private contractors and high-end groups are treated within the NHS. There's talk of training Tier 2 dentists as specialists in primary care, where the problems could be solved on the high street. The pressure would become enormous - high street dentistry is barely able to cope as it is. There are so many holes in the idea, and we will continue to voice our concerns in the strongest possible terms to ensure hospital dentists do not face these realities moving forward.'

Shawn suggests honest and open conversation is needed to move the agenda forward.

'What is urgently required now is two-fold. Firstly, honest and meaningful engagement now with myself and my BDA negotiating team to design and negotiate a reformed dental contract that can deliver NHS oral healthcare to our patients and create an environment in which dentists and their teams wish to work again.

'Secondly, a renewed and increased financial commitment by this Government to translate their good words into an NHS dental system that is fit for purpose, rather than the crisis laden system that currently exists. 
'The financial spend by Government on NHS dentistry is a third less in real terms than it was a decade ago; the single area of the NHS to have seen a decline in funding. As I said earlier, this crisis should be of no surprise to anyone and can be firmly laid at the door of Government. They have repeatedly ignored our calls for contract reform with the subsequent disastrous position that we and are patients now find ourselves.'

Paul also believes funding is a central pillar towards progress.

'Dental practice running costs are outstripping inflation by a significant percentage. In England there has been no funding for ventilation compared to that promised to the three other home nations and those practices who have yet to install methods of additional ventilation) other than opening a window welcome a degree of funding for the installation. The private sector self-funded this in order to be able to reduce the fallow time and increase their throughput of patients. Those practices who have funded these methods themselves should be reimbursed retrospectively.

'The profession needs to retain its present staff in both the private and NHS sectors - indeed the vast majority of practices do provide a percentage of both models of service delivery'.

Nikki also highlighted capacity issues, saying: 'We absolutely need to improve capacity to treat the patients that want to be treated. Recruitment problems aren't confined to dentistry, but they affect patients and in a healthcare profession, that's unacceptable.

'It's tied into the wider issues relating to pay and morale. If these were improved, the NHS would be a more attractive proposition, would retain more dentists and increase capacity. The Advancing Dental Care project is designed to improve pathways, but I don't see it working without Contract Reform happening.'

\section{Ramifications of no progress}

As always, change doesn't happen easily. It seems we've been discussing contract reform since the day the current contract began in 2006. In Northern Ireland, their lack of government stalled a new contract for the CDS. These examples aren't pointing fingers, but merely highlighting how difficult it is for dentistry to top the agenda. You have to ask why that is, especially when the pandemic highlighted the myriad of issues the profession has dealt with. According to Nikki, it begins at the top.
'Things have to change now, and others have mentioned there's an opportunity to do so', she suggested. 'Have we missed our window? I hope not. There's a lack of leadership in dentistry - too many dentists do not know where to look. The BDA has filled the void and called for clarity at every stage throughout the pandemic, and in many cases clarity and support simply hasn't been there.

Any changes that do happen will need to happen incrementally, and some things are easier to implement than others. It is abundantly clear, for example, that many NHS dentists are struggling to meet the $65 \%$ UDA threshold while high levels of infection prevention controls are still in place. Not being able to meet the needs of the population is not right, and something needs to change.'

\section{'Any changes that do happen will need to happen incrementally, and some things are easier to implement than others'}

Peter added: 'Right now I'd say we're at our weakest. Before long, the true impact of COVID-19 - on top of years of underfunding - will come to light, and my concern is this is in the pipeline. We need a review into dentistry now so we can finally address what the 'new norm' may be moving forwards.'

Shawn believes change needs to happen and now.

'These changes need to be implemented in 2022', he stated. 'This feels to me and the BDA team like a pivotal moment for NHS dentistry and its continued existence on any scale to meet the national patient need. We have been involved in a series of Advisory and Technical Working Groups with NHSE during the last six months and we are now moving to a 'design and negotiate' phase. Their engagement needs to be transparent, ambitious and focussed to deliver fundamental, funded reform. Without this the media headlines, MP letters and regular Parliamentary debates will continue and indeed worsen. That would continue to abjectly fail millions of patients, tax-payers and voters in this country.

'A continued delay will create further uncertainty for practices but of course the BDA will continue to offer advice and support to all members, in the difficult decisions that they have to make to ensure their futures. The time for ambitious dialogue and implementation by NHSE is now and we are prepared and ready to engage in that process.

Paul agrees immediate action is needed.

'I agree, Shawn. Changes need to be applied immediately and take into account the expenses practices have incurred over the past 18-20 months. The recruitment and retention of all dental personnel is at a crisis level. If this is not attended to the situation will be more even more acute and the practices who rely on the majority of their income stream will be left with no alternative but to increase their fees in order to offset their increasing costs. A tipping point may occur where private practices are providing care for those who can financially afford it.

'We must recruit and retain our colleagues, particularly newer graduates in the profession (the future of our industry) in the NHS sector or there is a real risk that they will be lost to the private sector. There is becoming a significant sea change in movement from NHS to private service delivery.'

Charlotte believes change is already afoot, and dentistry needs to ensure it is part of that.

'The changing landscape of the NHS in England is already being seen' she said. 'CDS will need to be agile and responsive, otherwise I fear we risk our services being forgotten or left behind. But CDS are excellent advocates and collaborators and I know they will ensure that the needs of our patients, services and workforce not forgotten during this time of change.'

To me, Charlotte's point about the changing NHS landscape is crucial to any successful improvement of dentistry. Too often dentistry has been left behind, and if she's right and change is already afoot, Nikki's belief that we may have already missed the boat is a pertinent one. The entire health service workforce across the four nations is exhausted, and dentists are no different. The exception is they have livelihoods to worry about - restrictions and lockdowns meant no and fewer patients, which impacts income. The impact for patients could be catastrophic and affect every service. Only time will tell how much of the backlog accrued throughout the pandemic can be cleared to the point we're back to pre-COVID-19 levels which, as pointed out above, were nothing to write home about. The vicious cycle dentistry finds itself stuck in, rather like Mr Creosote, is ready to blow.

https://doi.org/10.1038/s41404-021-0947-5 